\section{Novel Rapid Test for Detecting Carbapenemase}

\author{
Yanfang Feng, ${ }^{1}$ Akilan Palanisami, ${ }^{1}$ Jerrin Kuriakose, \\ Michael Pigula, Shoaib Ashraf, Tayyaba Hasan
}

Author affiliations: Massachusetts General Hospital and Harvard Medical School, Boston, Massachusetts, USA (Y. Feng,

A. Palanisami, J. Kuriakose, M. Pigula, S. Ashraf, T. Hasan); Harvard-MIT Health Sciences and Technology, Cambridge, Massachusetts, USA (T. Hasan)

DOI: https://doi.org/10.3201/eid2604.181655

We developed a carbapenemase test based on the ability of imipenem to inhibit noncarbapenemase $\beta$-lactamases. The test uses bacterial isolates with a fluorescent $\beta$-lactamase substrate, producing objective results with $100 \%$ sensitivity and specificity in 10 minutes. The assay is inexpensive and consists of only 1 mixing step.

A $\mathrm{s}$ a potent $\beta$-lactamase, carbapenemase can degrade almost all $\beta$-lactam antimicrobial drugs, including the carbapenems, regarded as the last line of therapy for many life-threatening infections $(1,2)$. Various epidemic types of carbapenemase have been reported globally, including Klebsiella pneumoniae carbapenemase, Verona integron-encoded metallo$\beta$-lactamase, Serratia marcescens enzyme, imipenem-hydrolyzing $\beta$-lactamase, New Delhi metallo$\beta$-lactamase, oxacillinase, metallo- $\beta$-lactamase, and São Paulo metallo- $\beta$-lactamase (1). If uncontrolled, the spread of these carbapenemases is expected to increase therapeutic failure and leave many patients with no effective treatment options.

Despite the urgency, timely carbapenemase detection remains a challenge for microbiology laboratories. Phenotypic assays are inexpensive and easily performed, but their use requires 24-48 hours and many lack sensitivity or specificity (3). The widespread use of other assays (e.g., molecular tests of carbapenemase genes, mass spectrometry detection of carbapenem hydrolysis) is impeded by the expertise required to perform them and their cost $(4,5)$. The recently developed (2012) Carba NP test and variants are elegant solutions, but their use requires up to 2 hours (6). Further improvements in test rapidity and simplicity are highly desirable, especially for patients in critical condition, who need immediate therapy and infection control action.

We demonstrate that by using fluorescence identification of $\beta$-lactamase activity (FIBA), carbapenemase production in bacteria can be detected

${ }^{1}$ These authors contributed equally to this article. sensitively and specifically in 10 minutes, with only 1 step. FIBA uses a dark fluorescence probe, $\beta$-LEAF ( $\beta$-lactamase enzyme-activated fluorophore), which turns fluorescent when cleaved by $\beta$-lactamases, including penicillinases, extendedspectrum $\beta$-lactamases (ESBL), AmpC $\beta$-lactamases, and carbapenemases $(7,8)$. Thus, the rate of fluorescence increase (hereafter called increase rate) is a measure of the bacterial $\beta$-lactamase activity and is reduced as the $\beta$-lactamase activity is hampered. For a noncarbapenemase $\beta$-lactamase, the increase rate will be reduced by the addition of imipenem, which binds the enzyme active site and blocks $\beta$-LEAF access (2). In contrast, the increase rate for a carbapenemase is relatively unaffected by imipenem addition because carbapenemase is able to rapidly cleave the imipenem and relieve the inhibition (1). Accordingly, bacteria that produce carbapenemases can be detected by comparing the increase rate with and without imipenem (Figure; Appendix Figure 1, https:/ / wwwnc.cdc.gov/EID/ article/26/4/18-1655-App1.pdf).

FIBA is performed in a 96-well plate. Each well contains $50 \mu \mathrm{L}$ of $20 \mu \mathrm{mol} / \mathrm{L} \beta$-LEAF, $25 \mu \mathrm{L}$ of phosphate-buffered saline with or without $40 \mu \mathrm{mol} / \mathrm{L}$ imipenem (Cayman Chemical, https://www.caymanchem.com), and $10 \mu \mathrm{L}$ of either $1 \mathrm{mg} / \mathrm{mL}$ polymyxin B nonapeptide or 1\% 3-[(3-cholamidopropyl) dimethylammonio]-1-propanesulfonate (Sigma-Aldrich, https://www.sigmaaldrich.com), which act as weak or strong permeabilizers, respectively. To start the assay, $25 \mu \mathrm{L}$ of $1 \times 10^{10} \mathrm{CFU} / \mathrm{mL}$ bacterial suspension made by colonies grown overnight on BHI agar (Sigma-Aldrich) is added to each well. To monitor the increase rate, fluorescence measurement is then performed at $37^{\circ} \mathrm{C}$ at 10 -s intervals for 10 min with Ex/Em 450/510 nm in the plate reader (Spectramax M5 plate reader, Molecular Devices, https://www.moleculardevices.com). For each bacterial sample, we performed the reactions in duplicate and averaged the results. We objectively interpreted the fluorescence measurements by using an automated Python script (Appendix), which required a few seconds after assay completion.

We tested FIBA on 76 randomly selected infection isolates from either the Centers for Disease Control and Prevention (9) or the American Type Culture Collection (https://www.atcc.org). The MICs of these isolates, if not predetermined, were measured by the 2017 Clinical Laboratory and Standards Institute (https://clsi.org) broth dilution method. Genetic test results for $\beta$-lactam resistance were provided with the isolates. Among these, 55 

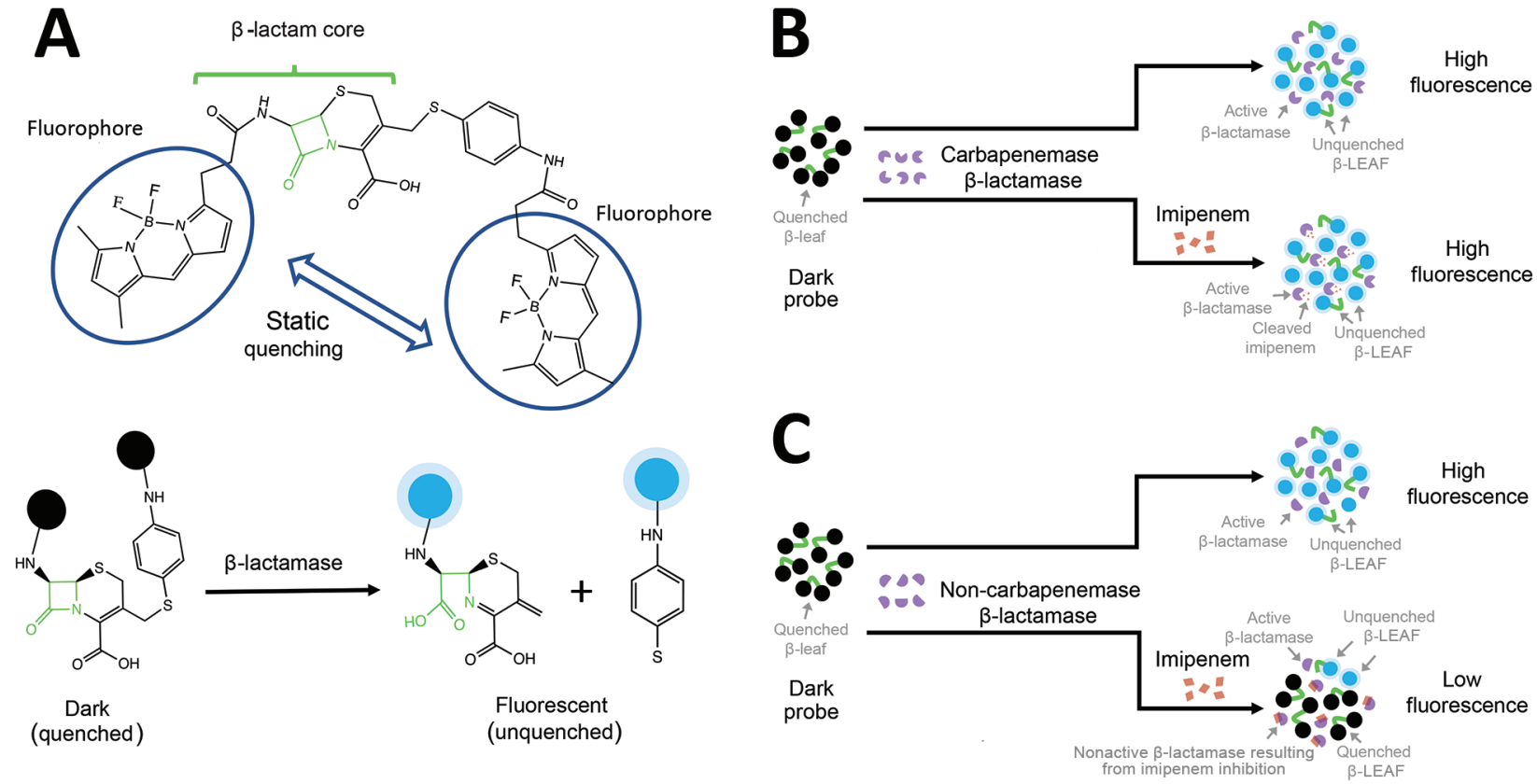

Figure. Schematic illustration of the principle of fluorescence identification of $\beta$-lactamase activity. A). The $\beta$-lactamase-activated fluorophore probe comprises a cleavable $\beta$-lactam core conjugated to 2 fluorophores (circled) that are quenched because of close proximity. This construct was designed to mimic the enzymatic degradation properties of easily cleavable $\beta$-lactam antimicrobial drugs. When this probe is attacked by $\beta$-lactamase, the probe core is cleaved, leading to the separation of the fluorophores and the recovery of their fluorescent properties (fluorescent state). B) Assay profile for carbapenemase-producing bacteria.

C) Assay profile for non-carbapenemase-producing bacteria. Black, quenched fluorophore; blue, unquenched fluorophore turning fluorescent; green, $\beta$-lactam core; red, imipenem; purple, $\beta$-lactamase. $\beta$-LEAF, $\beta$-lactamase enzyme-activated fluorophore.

were carbapenemase positive, carrying the major epidemic carbapenemase types including K. pneumoniae carbapenemase $(\mathrm{n}=20)$, imipenem-hydrolyzing $\beta$-lactamase $(n=2)$, metallo- $\beta$-lactamase $(n$ $=4)$, New Delhi metallo- $\beta$-lactamase $(\mathrm{n}=10)$, oxacillinase $(n=8), S$. marcescens enzyme $(n=2)$, São Paulo metallo- $\beta$-lactamase $(n=1)$, Verona integronencoded metallo- $\beta$-lactamase $(n=6)$, and New Delhi metallo- $\beta$-lactamase oxacillinase $(n=2)$. The other 21 isolates expressed noncarbapenemase $\beta$-lactamases, which involved 9 isolates with only ESBL, 3 isolates with both ESBL and porin modification, 6 isolates with only AmpC $\beta$-lactamase, and 3 isolates with both ESBL and AmpC $\beta$-lactamase. Among these isolates, 3 were carbapenem resistant. The entire panel, which included 28 colistin-resistant strains (MIC $\geq 4 \mathrm{ug} / \mathrm{mL}$ ), was classified successfully with FIBA (Appendix Tables 1, 2), resulting in 100\% sensitivity $(95 \%$ CI $94 \%-100 \%)$ and $100 \%$ specificity (95\% CI 84\%-100\%).

The primary limitation of this study is the small number of isolates evaluated. However, the breadth of isolates studied here included 8 enzyme types across 16 species, suggesting the generality of the approach.
FIBA can be performed $\approx 10$ times faster than the most rapid carbapenemase test commercially available while maintaining comparable sensitivity and specificity $(6,10)$. Its automated analysis improves turnaround time and reduces operator variability. With a reagent cost/assay of $\approx$ US $\$ 1$, FIBA is close in price to phenotypic tests but substantially faster and less labor intensive. Furthermore, the FIBA paradigm is extensible; by replacing imipenem with other known subtype-dependent inhibitors of carbapenemase (e.g., clavulanic acid, EDTA), rapid carbapenemase subtyping may also be possible. Our study demonstrates that low-cost, rapid assessment of carbapenemase can be performed in a 1-step format suitable for large-scale epidemiologic studies, thereby providing a new tool for infection outbreak control.

\section{Acknowledgments}

We acknowledge Gerry Nau for helpful conversations.

The research was supported by NIH/FIC R21TW010202 and DOD/AFOSR FA9550-16-1-0479. Patent pending for the $\beta$-LEAF design (application no. US20180094292A1). 


\section{About the Author}

Dr. Feng is a research fellow at Massachusetts General Hospital and Harvard Medical School, Boston. Her major research interests are molecular mechanisms, epidemiology, and prevention of drug resistance in bacteria.

\section{References}

1. Queenan AM, Bush K. Carbapenemases: the versatile beta-lactamases. Clin Microbiol Rev. 2007;20:440-58. https://doi.org/10.1128/CMR.00001-07

2. Papp-Wallace KM, Endimiani A, Taracila MA, Bonomo RA. Carbapenems: past, present, and future. Antimicrob Agents Chemother. 2011;55:4943-60. https:/ / doi.org/10.1128/ AAC.00296-11

3. Tamma PD, Opene BN, Gluck A, Chambers KK, Carroll KC, Simner PJ. Comparison of 11 phenotypic assays for accurate detection of carbapenemase-producing Enterobacteriaceae. J Clin Microbiol. 2017;55:1046-55. https:/ / doi.org/10.1128/ JCM.02338-16

4. Lasserre C, De Saint Martin L, Cuzon G, Bogaerts P, Lamar E, Glupczynski Y, et al. Efficient detection of carbapenemase activity in Enterobacteriaceae by matrixassisted laser desorption ionization-time of flight mass spectrometry in less than 30 minutes. J Clin Microbiol. 2015;53:2163-71. https://doi.org/10.1128/JCM.03467-14

5. Monteiro J, Widen RH, Pignatari AC, Kubasek C, Silbert S. Rapid detection of carbapenemase genes by multiplex real-time PCR. J Antimicrob Chemother. 2012;67:906-9. https://doi.org/10.1093/jac/dkr563

6. Nordmann P, Poirel L, Dortet L. Rapid detection of carbapenemase-producing Enterobacteriaceae. Emerg Infect Dis. 2012;18:1503-7. https://doi.org/10.3201/ eid1809.120355

7. Sallum UW, Zheng X, Verma S, Hasan T. Rapid functional definition of extended spectrum $\beta$-lactamase activity in bacterial cultures via competitive inhibition of fluorescent substrate cleavage. Photochem Photobiol. 2010;86:1267-71. https:/ / doi.org/10.1111/j.1751-1097.2010.00801.x

8. Khan S, Sallum UW, Zheng X, Nau GJ, Hasan T. Rapid optical determination of $\beta$-lactamase and antibiotic activity. BMC Microbiol. 2014;14:84. https:/ / doi.org/10.1186/ 1471-2180-14-84

9. CDC and FDA Antibiotic Resistance Isolate Bank [cited 2019 Mar 8]. https:// wwwn.cdc.gov/ARIsolateBank

10. Maurer FP, Castelberg C, Quiblier C, Bloemberg GV, Hombach M. Evaluation of carbapenemase screening and confirmation tests with Enterobacteriaceae and development of a practical diagnostic algorithm. J Clin Microbiol. 2015;53:95-104. https:/ / doi.org/10.1128/ JCM.01692-14

Address for correspondence: Tayyaba Hasan, The Wellman Center for Photomedicine, Massachusetts General Hospital, 40 Blossom St, Boston, MA 02114, USA; email: thasan@mgh.harvard.edu

\section{Arthritis Caused by MRSA CC398 in Patient without Animal Contact, Japan}

\author{
Hidemasa Nakaminami, ${ }^{1}$ Yuji Hirai, ${ }^{1}$ Hirosuke \\ Nishimura, Shunsuke Takadama, Norihisa Noguchi
}

\begin{abstract}
Author affiliations: Tokyo University of Pharmacy and Life
Sciences, Tokyo, Japan (H. Nakaminami, S. Takadama, N. Noguchi); Tokyo Medical University Hachioji Medical Center, Tokyo (Y. Hirai, H. Nishimura)
\end{abstract}

\section{DOI: https://doi.org/10.3201/eid2604.190376}

Clonal complex 398 methicillin-resistant Staphylococcus aureus (MRSA) is a typical lineage of livestock-associated MRSA. We report a case of intractable arthritis of the shoulder joint caused by a multidrug-resistant Panton-Valentine leukocidin-positive livestock-associated MRSA clonal complex 398 sequence type 1232 clone in a patient in Japan who had no animal contact.

Tn the past decade, methicillin-resistant Staphylococcus aureus (MRSA) has been detected in livestock, including swine, poultry, and veal calves $(1,2)$. In general, the virulence of animal-derived livestock-associated (LA-MRSA) strains is considered to be lower than that of community-acquired MRSA lineages (3). However, LA-MRSA strains can effectively colonize and infect humans, with subsequent transmission in both community and hospital settings. Human colonization with LAMRSA sequence type (ST) 398 was first recognized among swine farmers in France and the Netherlands in the early 2000s (4). According to Larsen et al., clonal complex (CC) 398 MRSA accounted for $21 \%$ of MRSA isolated from skin and soft tissue infections in Denmark during 2010-2015 (5). However, ST398 MRSA has not been isolated in patients in Japan. We report a case of intractable arthritis of the shoulder joint caused by a multidrug-resistant Panton-Valentine leukocidin (PVL)-positive LAMRSA CC398 (ST1232) clone in a patient in Japan who had no animal contact.

We performed MRSA identification, staphylococcal cassette chromosome (SCC) mec typing, spa typing, multilocus sequence typing (MLST), MIC determination, and PCR assays for detecting virulence factors and antimicrobial resistance genes, as described previously $(1,6)$. The study protocol was approved by the Tokyo University of Pharmacy and Life Sciences Ethics Committee (approval no. 12-09). 